\title{
О ЗАДАЧЕ ПОСЛЕДОВАТЕЛЬНОГО ОБХОДА МЕГАПОЛИСОВ С УСЛОВИЯМИ ПРЕДШЕСТВОВАНИЯ И ФУНКЦИЯМИ СТОИМОСТИ С ЗАВИСИМОСТЬЮ ОТ СПИСКА ЗАДАНИЙ ${ }^{1}$
}

\begin{abstract}
А. Г. Ченцов, А. А. Ченцов, А. Н. Сесекин
Исследуется решение задачи маршрутизации перемещений с ограничениями и усложненными функциями стоимости. Более того, построение последних может быть затруднено, а потому этапы данного построения являются элементами решения задачи. Такая ситуация складывается, в частности, при исследовании инженерной задачи о демонтаже радиационно опасных элементов, где в рамках традиционной для дискретной оптимизации постановки требуется недопустимо большое время для построения матрицы затрат, элементы которой характеризуют дозы радиации, получаемые исполнителями на этапе перемещений и работ по демонтажу. Допускается, что на этапе вычислительной реализации получаемого оптимального алгоритма соответствующие "части" матрицы могут и не заноситься в память компьютера, а вычисляться по мере надобности. Применение развиваемых методов может быть связано с задачей демонтажа энергоблока АЭС, выведенного из эксплуатации.
\end{abstract}

Ключевые слова: динамическое программирование, маршрут, функция Беллмана.

A. G. Chentsov, A. A. Chentsov, A. N. Sesekin. On the problem of sequential traversal of megalopolises with precedence conditions and cost functions depending on a list of tasks.

A constrained routing problem with complicated cost functions is studied. The construction of the cost functions can be difficult, and therefore the stages of this construction are elements of the solution of the problem. This situation arises, in particular, in studying the engineering problem of dismantling radiation hazardous elements, where, in the framework of a problem statement traditional for discrete optimization, it takes an unacceptably long time to construct a cost matrix whose entries characterize the radiation doses received by performers at the stage of displacement and dismantling. It is assumed that, at the stage of the computational implementation of the resulting optimal algorithm, the corresponding "parts" of the matrix may be not fed to the computer's memory but calculated as needed. Possible applications of the developed methods may be related to the problem of dismantling a decommissioned generator unit of an NPP.

Keywords: dynamic programming, route, Bellman function.

MSC: 05A05, 97N70, 97N80

DOI: $10.21538 / 0134-4889-2020-26-3-219-234$

\section{Введение}

Проблема маршрутизации, возникающая в инженерных задачах, имеет своим прототипом известную труднорешаемую задачу коммивояжера (3К или TSP в англоязычной литературе; см. [1-9]), однако обладает и целым рядом особенностей не только количественного, но и качественного характера. Среди данных особенностей отметим сейчас ограничения различных типов и усложненные функции стоимости. Последние могут при этом не задаваться непосредственно, а должны рассчитываться в процессе решения задачи, причем соответствующие процедуры оказываются зачастую весьма трудоемкими. Особенно серьезные требования (по совокупности причин) предъявляются к ресурсам памяти вычислителя.

В настоящем исследовании мы ориентируемся на применения, связанные с демонтажем радиационно опасных элементов. В этих инженерных задачах функции (а на самом деле матрицы) затрат реально включают зависимость от списка заданий. Речь идет о проблеме снижения дозовой нагрузки исполнителей при реализации комплекса заданий по демонтажу.

\footnotetext{
${ }^{1}$ Работа выполнена при поддержке РФФИ (проект 19-01-00573) и в рамках исследований, проводимых в Уральском математическом центре.
} 
Тогда исполнитель в каждый момент времени находится под радиационным воздействием тех и только тех источников, которые еще не демонтированы. Отсюда и возникает зависимость функций стоимости от списка невыполненных заданий [10, гл. 2]. Помимо этого имеются и естественные ограничения, среди которых отметим сейчас условия предшествования (см. [4;11-13]). Так, например, одни излучающие элементы могут располагаться на других, образуя своеобразный верхний ярус; поэтому "верхние" элементы должны демонтироваться раньше.

Мы используем ниже модель мегаполисов, понимая под мегаполисами непустые конечные множества, являющиеся объектами посещений. В качестве таковых могут использоваться дискретизированные (в целях компьютерных вычислений) границы ближних зон источников или системы входов-выходов в помещениях, в которых располагаются соответствующие источники. Исполнитель может в последнем случае выбрать допустимый вход в помещение, переместиться к источнику, провести работы по его демонтажу (это может потребовать определенного времени), после чего переместиться к выходу и покинуть помещение; точки входа и выхода исполнитель выбирает сам (в случае, когда мегаполис возникает в результате дискретизации ближней зоны "открытого" источника, смысл выполняемых операций аналогичен). Упомянутые выше этапы, связанные с посещением мегаполиса, будем связывать с выполнением внутренних (по отношению к мегаполису) работ. Упомянутые совокупности внутренних работ "соединяются" в целое посредством внешних перемещений (между мегаполисами, а также из начального состояния - точки старта - к первому в смысле выбранной очередности мегаполису). Точка старта также выбирается исполнителем в пределах заданного множества.

Исследуемая задача существенно отличается, как видно из вышеупомянутого описания, от 3К; она является значительно более трудной и в количественном, и в качественном отношении. В этой связи требуется достаточно развитая теоретическая конструкция, включая элементы формализации. Излагаемые ниже построения соответствуют работе [14]. Мы рассматриваем в качестве решения любой триплет, включающий точку старта, перестановку индексов, характеризующую очередность посещений мегаполисов и конкретную траекторию посещения (занумерованных) мегаполисов. Такую перестановку индексов называем маршрутом, следуя традиции ЗК. В качестве критерия качества в конкретной постановке задачи мы будем использовать суммарную дозу радиации, получаемую исполнителем при внешних перемещениях и внутренних работах. Для определения упомянутых доз используются процедуры интегрирования нелинейных зависимостей вдоль траектории исполнителя.

В качестве основного метода исследования принят аппарат динамического программирования (ДП) в реализации, которая развивает схему Р. Беллмана (см. [7]; в дискретной оптимизации для решения задач типа ЗК более широко используется схема Хелда - Карпа, см. [8]). Данная реализация восходит к $[15, \S 4.9]$ (см. также [16]). В этой реализации метода ДП существенно используются условия предшествования, позволяющие несколько снизить вычислительную сложность. В рамках предполагаемой конструкции (как и в [15; 16]) при наличии условий предшествования не производится построение всего массива значений функции Беллмана; вместо этого определяются посредством рекуррентной процедуры слои упомянутой функции, что приводит к меньшему объему вычислений и экономии памяти.

В связи с применением методов маршрутизации к задачам атомной энергетики, связанных с минимизацией дозовой нагрузки, отметим наряду с [10], работы [16-20]), а также [21], где рассматривалась несколько иная маршрутная задача, связанная с перемещением тепловыделяющих сборок.

Разрабатываемые авторами методы могут найти и находят применение в различных инженерных задачах маршрутной оптимизации, осложненных нестандартными ограничениями. Например, содержательные инженерные задачи, связанные с оптимизацией движения режущего инструмента на машинах с ЧПУ, для которых применимы такие методы решения, рассматриваются в [22-29]. 


\section{1. Сведения общего характера}

Используется стандартная теоретико-множественная символика (кванторы, пропозициональные связки и др.); через $\varnothing$ обозначаем пустое множество, $\triangleq-$ равенство по определению, def заменяет фразу "по определению". Семейством называем множество, все элементы которого сами являются множествами. Любым двум объектам $x$ и $y$ сопоставляется неупорядоченная пара $\{x ; y\}$ этих объектов, т. е. единственное множество, содержащее $x, y$ и не включающее никаких других элементов. Соответственно каждому объекту $z$ сопоставляется синглетон $\{z\} \triangleq\{z ; z\}$, содержащий $z: z \in\{z\}$. Каждое множество - объект. Поэтому, следуя [30, с. 67], двум произвольным объектам $u$ и $v$ сопоставляем упорядоченную пару (УП) $(u, v) \triangleq\{\{u\} ;\{u ; v\}\}$ с первым объектом $u$ и вторым объектом $v$. В свою очередь, произвольной УП $h$ сопоставляем ее первый элемент $p r_{1}(h)$ и второй элемент $p r_{2}(h)$, однозначно определяемые равенством $h=\left(p r_{1}(h), p r_{2}(h)\right)$. Если $x, y$ и $z$ - объекты, то $(x, y, z) \triangleq((x, y), z)$ есть триплет (см. [31, с. 17]) с первым элементом $x$, вторым элементом $y$ и третьим элементом $z$.

Каждому множеству $S$ сопоставляем семейство $\mathcal{P}(S)$ всех подмножеств (п/м) $S$, тогда $\mathcal{P}^{\prime}(S) \triangleq \mathcal{P}(S) \backslash\{\varnothing\}$ есть семейство всех непустых п/м $S$, а $\operatorname{Fin}(S)$ - семейство всех непустых конечных п/м $S$ (ясно, что $\left.\operatorname{Fin}(S) \subset \mathcal{P}^{\prime}(S)\right)$. Если же $S$ - конечное множество, то $\operatorname{Fin}(S)=$ $\mathcal{P}^{\prime}(S)$. Используем стандартное определение декартова произведения двух множеств. Если же $A, B$ и $C$ - три множества, то, как обычно, полагаем, что (см. [31, с. 17]) $A \times B \times C \triangleq(A \times B) \times C$; это согласуется с вышеупомянутым определением триплета.

Если $X$ и $Y$ - непустые множества, то через $Y^{X}$ обозначается множество всех отображений из $X$ в $Y$; тогда выражения

$$
\mathbf{f} \in Y^{X} \quad \text { и } \quad \mathbf{f}: X \longrightarrow Y
$$

отождествимы; при $g \in Y^{X}$ и $x \in X$ в виде $g(x), g(x) \in Y$, имеем значение отображения $g$ в точке $x$. Для непустых множеств $A, B, C \in \mathcal{P}^{\prime}(B)$ и отображения $h \in B^{A}$ в виде $h^{1}(C) \triangleq\{h(x): x \in C\} \in \mathcal{P}^{\prime}(B)$ имеем образ множества $C$ при действии $h$. Как обычно, для непустых множеств $A, B, D$, отображения $\varphi \in D^{A \times B}$, а также точек $a \in A$ и $b \in B$ полагаем $\varphi(a, b) \triangleq \varphi((a, b))$, получая значение $\varphi$ в точке $(a, b)$. Если же $X, Y, Z, T-$ непустые множества, $\psi \in T^{X \times Y \times Z}, s \in X \times Y$ и $l \in Z$, то определено значение $\psi(s, l)$ отображения $\psi$ в точке $(s, l)=\left(\left(p r_{1}(s), p r_{2}(s)\right), l\right)$; для этого значения используем также соглашение $\psi\left(p r_{1}(s), p r_{2}(s), l\right) \triangleq \psi(s, l) \in T$. Отображения такого типа понадобятся, в частности, в случае $T=\mathbb{R}$, где $\mathbb{R}$ есть (здесь и ниже) вещественная прямая.

Полагаем, что $\mathbb{R}_{+} \triangleq\{\xi \in \mathbb{R} \mid 0 \leq \xi\}, \mathbb{N} \triangleq\{1 ; 2 ; \ldots\}$ (натуральный ряд), $\mathbb{N}_{0} \triangleq\{0\} \cup \mathbb{N}=$ $\{0 ; 1 ; 2 ; \ldots\} \in \mathcal{P}^{\prime}\left(\mathbb{R}_{+}\right)$и $\overline{p, q} \triangleq\left\{k \in \mathbb{N}_{0} \mid(p \leq k) \&(k \leq q)\right\}$ есть при $p \in \mathbb{N}_{0}$ и $q \in \mathbb{N}_{0}$ обычный порядковый интервал в $\mathbb{N}_{0}$; случай $\overline{p, q}=\varnothing$ допускается (при $q<p$ ). Ясно, что $\overline{1, n}=\{k \in$ $\mathbb{N} \mid k \leq n\} \forall n \in \mathbb{N}$.

Мы полагаем, что $\mathcal{R}_{+}[S] \triangleq\left(\mathbb{R}_{+}\right)^{S}$ для всякого непустого множества $S$, получая множество всех неотрицательных в/з функций на $S$. Если $K$ есть непустое конечное множество, то через $|K|$ обозначаем мощность $K,|K| \in \mathbb{N}$; полагаем также, что $|\varnothing| \triangleq 0$. Для непустого конечного множества $K$ через $(b i)[K]$ обозначаем множество всех биекций "интервала" $\overline{1,|K|}$ на $K$. В частности, мы можем исследовать $(b i)[\overline{1, n}]$ при $n \in \mathbb{N}$. Используемые ниже кортежи рассматриваются как функции на конечном п/м $\mathbb{N}$. При этом часто используется индексная форма записи: если $n \in \mathbb{N}$ и $H-$ непустое множество, в котором выделены точки $h_{1} \in H, \ldots, h_{n} \in H$, то $\left(h_{i}\right)_{i \in \overline{1, n}}$ следует понимать как отображение $\mathbf{h}: \overline{1, n} \longrightarrow H$, для которого $\mathbf{h}(j) \triangleq h_{j} \forall j \in \overline{1, n}$. Для таких отображений применимо, конечно, понятие образа непустого п/м $\overline{1, n}$ : при $K \in \mathcal{P}^{\prime}(\overline{1, n})$ имеем $\mathbf{h}^{1}(K)=\left\{h_{j}: j \in K\right\} \in \mathcal{P}^{\prime}(H)$.

\section{2. Постановка задачи (общий случай)}

В дальнейшем фиксируем непустое множество $X$ и его (непустое конечное) п/м $X^{0} \in$ $\operatorname{Fin}(X)$. Элементы $X^{0}$ рассматриваем как возможные точки старта. Пусть $N \in \mathbb{N}$ и $N \geq 2$. 
Каждому индексу из $\overline{1, N}$ сопоставлено непустое конечное п/м $X$ - мегаполис. Таким образом,

$$
M_{1} \in \operatorname{Fin}(X), \ldots, M_{N} \in \operatorname{Fin}(X)
$$

есть набор мегаполисов, являющихся объектами посещения. Следуя [15-20], полагаем, что

$$
\left(X^{0} \cap M_{j}=\varnothing \forall j \in \overline{1, N}\right) \&\left(M_{p} \cap M_{q}=\varnothing \forall p \in \overline{1, N} \forall q \in \overline{1, N} \backslash\{p\}\right) .
$$

Условия (2.1) типичны для задач маршрутизации. Фиксируем непустые отношения

$$
\mathbb{M}_{1} \in \mathcal{P}^{\prime}\left(M_{1} \times M_{1}\right), \ldots, \mathbb{M}_{N} \in \mathcal{P}^{\prime}\left(M_{N} \times M_{N}\right) .
$$

В связи с $(2.2)$ отметим, полагая $\mathbb{P} \triangleq(b i)[\overline{1, N}]$, что при $j \in \overline{1, N}$

$$
\left(\mathfrak{M}_{j} \triangleq\left\{p r_{1}(z): z \in \mathbb{M}_{j}\right\} \in \operatorname{Fin}\left(M_{j}\right)\right) \&\left(\mathbf{M}_{j} \triangleq\left\{p r_{2}(z): z \in \mathbb{M}_{j}\right\} \in \operatorname{Fin}\left(M_{j}\right)\right) .
$$

В дальнейшем изучаем вопросы организации перемещений следующего типа:

$$
\left(x \in X^{0}\right) \longrightarrow\left(x_{1}^{(1)} \in \mathfrak{M}_{\alpha(1)} \rightsquigarrow x_{2}^{(1)} \in \mathbf{M}_{\alpha(1)}\right) \longrightarrow \ldots \longrightarrow\left(x_{1}^{(N)} \in \mathfrak{M}_{\alpha(N)} \rightsquigarrow x_{2}^{(N)} \in \mathbf{M}_{\alpha(N)}\right),
$$

где $\alpha \in \mathbb{P}$; при этом постулируем, что $\left(x_{1}^{(1)}, x_{2}^{(1)}\right) \in \mathbb{M}_{\alpha(1)}, \ldots,\left(x_{1}^{(N)}, x_{2}^{(N)}\right) \in \mathbb{M}_{\alpha(N)}$. Разумеется, при последних условиях вместо (2.4) можно рассматривать модель перемещений следующего вида:

$$
\left((x, x) \in X^{0} \times X^{0}\right) \longrightarrow\left(z^{(1)} \in \mathbb{M}_{\alpha(1)}\right) \longrightarrow \ldots \longrightarrow\left(z^{(N)} \in \mathbb{M}_{\alpha(N)}\right) .
$$

Ниже будет приведено точное описание множества перемещений (2.5) при фиксации $\alpha \in \mathbb{P}$. Сейчас отметим, что

$$
\left(\mathbb{X} \triangleq\left(\bigcup_{i=1}^{N} \mathfrak{M}_{i}\right) \bigcup X^{0} \in \operatorname{Fin}(X)\right) \&\left(\mathbf{X} \triangleq\left(\bigcup_{i=1}^{N} \mathbf{M}_{i}\right) \bigcup X^{0} \in \operatorname{Fin}(X)\right) .
$$

Мы рассматриваем $\mathbb{X} \times \mathbf{X}$ как (непустое конечное) фазовое пространство процессов типа (2.4), (2.5). Для описания последних введем при $\alpha \in \mathbb{P}$ и $x \in X^{0}$ множество

$$
\mathcal{Z}_{\alpha}[x] \triangleq\left\{\left(z_{t}\right)_{t \in \overline{0, N}} \in \mathbb{Z} \mid\left(z_{0}=(x, x)\right) \&\left(z_{s} \in \mathbb{M}_{\alpha(s)} \forall s \in \overline{1, N}\right)\right\} \in \operatorname{Fin}(\mathbb{Z}),
$$

где $\mathbb{Z}$ есть def множество всех отображений из $\overline{0, N}$ в $\mathbb{X} \times \mathbf{X}$, т. е.

$$
\mathbb{Z} \triangleq(\mathbb{X} \times \mathbf{X})^{\overline{0, N}}
$$

(напомним, что $X^{0} \times X^{0}-$ непустое конечное множество). Для введения условий предшествования фиксируем множество $\mathbf{K} \in \mathcal{P}(\overline{1, N} \times \overline{1, N})$, для которого

$$
\forall \mathbf{K}_{0} \in \mathcal{P}^{\prime}(\mathbf{K}) \exists z_{0} \in \mathbf{K}_{0}: p r_{1}\left(z_{0}\right) \neq p r_{2}(z) \forall z \in \mathbf{K}_{0}
$$

(в [15] указаны содержательные примеры выполнения (2.7)). Тогда (при условии (2.7)) получаем (непустое конечное; см. [15, (2.2.53)]) множество

$$
\begin{gathered}
\mathbf{A} \triangleq\left\{\alpha \in \mathbb{P} \mid \forall t_{1} \in \overline{1, N} \forall t_{2} \in \overline{1, N}\left(\left(\alpha\left(t_{1}\right), \alpha\left(t_{2}\right)\right) \in \mathbf{K}\right) \Rightarrow\left(t_{1}<t_{2}\right)\right\} \\
=\left\{\alpha \in \mathbb{P} \mid \alpha^{-1}\left(\operatorname{pr}_{1}(z)\right)<\alpha^{-1}\left(p r_{2}(z)\right) \forall z \in \mathbf{K}\right\} \in \mathcal{P}^{\prime}(\mathbb{P})
\end{gathered}
$$

всех $\mathbf{K}$ - допустимых (допустимых по предшествованию) маршрутов. Если $x \in X^{0}$ и $\alpha \in \mathbf{A}$, то определено (см. (2.6)) множество $\mathcal{Z}_{\alpha}[x] \in \operatorname{Fin}(\mathbb{Z})$ - пучок траекторий, возможных при старте из $x$ и фиксации маршрута $\alpha$. Мы имеем при этом в виде

$$
\widetilde{\mathbf{D}}[x] \triangleq\left\{\left(\alpha,\left(z_{t}\right)_{t \in \overline{0, N}}\right) \in \mathbf{A} \times \mathbb{Z} \mid\left(z_{t}\right)_{t \in \overline{0, N}} \in \mathcal{Z}_{\alpha}[x]\right\} \in \operatorname{Fin}(\mathbf{A} \times \mathbb{Z}),
$$


где $x \in X^{0}$, множество всех допустимых решений (ДР) в случае, когда точка старта фиксирована. Соответственно

$$
\mathbf{D} \triangleq\left\{\left(\alpha,\left(z_{t}\right)_{t \in \overline{0, N}}, x\right) \in \mathbf{A} \times \mathbb{Z} \times X^{0} \mid\left(\alpha,\left(z_{t}\right)_{t \in \overline{0, N}}\right) \in \widetilde{\mathbf{D}}[x]\right\} \in \operatorname{Fin}\left(\mathbf{A} \times \mathbb{Z} \times X^{0}\right)
$$

есть множество всех ДР формулируемой ниже полной задачи. Для введения последней полагаем заданными функции

$$
\mathbf{c} \in \mathcal{R}_{+}[\mathbf{X} \times \mathbb{X} \times \mathfrak{N}], \quad c_{1} \in \mathcal{R}_{+}\left[\mathbb{M}_{1} \times \mathfrak{N}\right], \ldots, c_{N} \in \mathcal{R}_{+}\left[\mathbb{M}_{N} \times \mathfrak{N}\right], \quad f \in \mathcal{R}_{+}\left[\bigcup_{i=1}^{N} \mathbf{M}_{i}\right]
$$

где $\mathfrak{N} \triangleq \mathcal{P}^{\prime}(\overline{1, N})$. Функция с необходима для оценивания внешних перемещений (между мегаполисами, а также между точкой из $X^{0}$ и соответствующим мегаполисом). Значения функции $c_{j}$, где $j \in \overline{1, N}$, используются для оценивания работ, связанных с посещением мегаполиса $M_{j}$ и именуемых далее внутренними, а функция $f$ - при оценивании терминального состояния (точка $x_{2}^{(N)}$ в (2.4)). Если $x \in X^{0}, \alpha \in \mathbb{P}$ и $\left(z_{t}\right)_{t \in \overline{0, N}} \in \mathcal{Z}_{\alpha}[x]$, то определено значение

$$
\mathfrak{G}_{\alpha}\left[\left(z_{t}\right)_{t \in \overline{0, N}}\right] \triangleq \sum_{t=1}^{N}\left[\mathbf{c}\left(p r_{2}\left(z_{t-1}\right), p r_{1}\left(z_{t}\right), \alpha^{1}(\overline{t, N})\right)+c_{\alpha(t)}\left(z_{t}, \alpha^{1}(\overline{t, N})\right)\right]+f\left(p r_{2}\left(z_{N}\right)\right) \in \mathbb{R}_{+}
$$

для наших целей существенным является случай, когда $\alpha \in \mathbf{A}$. Тогда при $x \in X^{0}$ имеем задачу

$$
\mathfrak{G}_{\alpha}\left[\left(z_{t}\right)_{t \in \overline{0, N}}\right] \longrightarrow \min , \quad\left(\alpha,\left(z_{t}\right)_{t \in \overline{0, N}}\right) \in \widetilde{\mathbf{D}}[x] .
$$

Для нее определены экстремум $\widetilde{V}[x]$ в виде наименьшего из чисел $\mathfrak{G}_{\alpha}\left[\left(z_{t}\right)_{t \in \overline{0, N}}\right],\left(\alpha,\left(z_{t}\right)_{t \in \overline{0, N}}\right) \in$ $\widetilde{\mathbf{D}}[x]$, и (непустое конечное) множество

$$
(\operatorname{sol})[x] \triangleq\left\{\left(\alpha^{0},\left(z_{t}^{0}\right)_{t \in \overline{0, N}}\right) \in \widetilde{\mathbf{D}}[x] \mid \mathfrak{G}_{\alpha^{0}}\left[\left(z_{t}^{0}\right)_{t \in \overline{0, N}}\right]=\widetilde{V}[x]\right\} \in \mathcal{P}^{\prime}(\widetilde{\mathbf{D}}[x])
$$

всех оптимальных решений этой задачи. Кроме того, отметим, что (согласно (2.8)) при $x \in X^{0}$ и $\left(\alpha,\left(z_{t}\right)_{t \in \overline{0, N}}, x\right) \in \mathbf{D}$ установлено (см. (2.10)) значение

$$
\mathfrak{G}_{\alpha}\left[\left(z_{t}\right)_{t \in \overline{0, N}}\right] \in \mathbb{R}_{+}
$$

С учетом этого введем в рассмотрение полную задачу

$$
\mathfrak{G}_{\alpha}\left[\left(z_{t}\right)_{t \in \overline{0, N}}\right] \longrightarrow \min , \quad\left(\alpha,\left(z_{t}\right)_{t \in \overline{0, N}}, x\right) \in \mathbf{D}
$$

для которой определены значение (экстремум)

$$
\mathbb{V} \triangleq \min _{\left(\alpha,\left(z_{t}\right)_{t \in \overline{0, N}}, x\right) \in \mathbf{D}} \mathfrak{G}_{\alpha}\left[\left(z_{t}\right)_{t \in \overline{0, N}}\right]=\min _{x \in X^{0}} \tilde{V}[x] \in \mathbb{R}_{+}
$$

и (непустое конечное) множество

$$
\mathbf{S O L} \triangleq\left\{\left(\alpha^{0},\left(z_{t}^{0}\right)_{t \in \overline{0, N}}, x^{0}\right) \in \mathbf{D} \mid \mathfrak{G}_{\alpha^{0}}\left[\left(z_{t}^{0}\right)_{t \in \overline{0, N}}\right]=\mathbb{V}\right\} \in \operatorname{Fin}(\mathbf{D})
$$

Нашей целью является поиск $\mathbb{V}$ (см. (2.13)) и какого-либо решения из множества (2.14). 


\section{3. Специфика функций стоимости}

В настоящем разделе мы введем некоторые естественные предположения относительно функций (2.9), отражающие специфику задачи демонтажа системы радиационно опасных элементов. Прежде всего полагаем выполненным свойство аддитивности $\mathbf{c}:$ при $x \in \mathbf{X}, y \in \mathbb{X}$ и $K \in \mathfrak{N}$

$$
\mathbf{c}(x, y, K)=\sum_{k \in K} \mathbf{c}(x, y,\{k\}) .
$$

Значения $\mathbf{c}(x, y,\{k\}), x \in \mathbf{X}, y \in \mathbb{X}$ и $k \in \overline{1, N}$ определяются выражениями, приведенными в работе [32]. Итак (см. (3.1)), дозы радиации, создаваемые отдельными источниками, суммируются, а стало быть, функция с полностью определяется набором функций $\mathbf{c}(\cdot, \cdot,\{k\}), k \in \overline{1, N}$.

Рассмотрим естественные представления для функций $c_{1}, \ldots, c_{N}$. Будем исходить при этом из представлений последних в виде суммы трех компонент, две из которых оценивают перемещения, связанные с соответствующим мегаполисом, а третья характеризует работы непосредственно по демонтажу. Мы исходим из того, что (см. (2.3)) при $j \in \overline{1, N}$ точки $\mathfrak{M}_{j}$ играют роль пунктов прибытия в мегаполис $M_{j}$, а точки $\mathbf{M}_{j}$ - роль пунктов отправления из $M_{j}$. Относительно $c_{j}$, где $j \in \overline{1, N}$, мы по аналогии с (3.1) полагаем выполненным свойство аддитивности: при $z \in \mathbb{M}_{j}$ и $K \in \mathfrak{N}$

$$
c_{j}(z, K)=\sum_{k \in K} c_{j}(z,\{k\})
$$

здесь, конечно, $c_{j}(z, K)=c_{j}\left(z_{1}, z_{2}, K\right)$ и при $k \in K c_{j}(z,\{k\})=c_{j}\left(z_{1}, z_{2},\{k\}\right)$, где $z_{1} \triangleq p r_{1}(z)$ и $z_{2} \triangleq p_{2}(z)$. Таким образом, и при оценивании внутренних работ получаемые исполнителем дозы полностью определяются функциями $c_{j}(\cdot,\{k\}), k \in K$. поэтому для конкретного представления всех значений (3.2) нам достаточно иметь значения

$$
c_{j}(z,\{k\}), \quad j \in \overline{1, N}, \quad z \in \mathbb{M}_{j}, \quad k \in \overline{1, N} .
$$

Условимся о следующих обозначениях: при $t \in \overline{1, N}$

$$
\bar{c}_{t}^{(1)} \triangleq\left(c_{t}^{(1)}(x,\{k\})\right)_{(x, k) \in \mathfrak{M}_{t} \times \overline{1, N}}, \bar{c}_{t}^{(2)} \triangleq\left(c_{t}^{(2)}(\{k\})\right)_{k \in \overline{1, N}}, \bar{c}_{t}^{(3)} \triangleq\left(c_{t}^{(3)}(y,\{k\})\right)_{(y, k) \in \mathbf{M}_{t} \times \overline{1, N}} ;
$$

ясно,что $\bar{c}_{t}^{(1)} \in \mathcal{R}_{+}\left[\mathfrak{M}_{t} \times \overline{1, N}\right], \bar{c}_{t}^{(2)} \in \mathcal{R}_{+}[\overline{1, N}]$ и $\bar{c}_{t}^{(3)} \in \mathcal{R}_{+}\left[\mathbf{M}_{t} \times \overline{1, N}\right]$. При описании значений (3.3) мы исходим из следующего предположения: при $j \in \overline{1, N}, z \in \mathbb{M}_{j}$ и $k \in \overline{1, N}$

$$
c_{j}(z,\{k\})=\bar{c}_{j}^{(1)}\left(p r_{1}(z), k\right)+\bar{c}_{j}^{(2)}(k)+\bar{c}_{j}^{(3)}\left(p r_{2}(z), k\right) .
$$

Условимся, что при $j \in \overline{1, N}$ функция $c_{j}^{(1)} \in \mathcal{R}_{+}\left[\mathfrak{M}_{j} \times \mathfrak{N}\right]$ характеризует суммарное радиационное воздействие соответствующих одноточечных источников на исполнителя при перемещении от пункта прибытия в мегаполис $M_{j}$ до места проведения работы по демонтажу, а функция $c_{j}^{(3)}$ оценивает аналогичное радиационное воздействие при перемещении от места проведения упомянутой работы до пункта отправления; значения функции $c_{j}^{(2)}$ характеризуют суммарное радиационное воздействие одиночных источников на исполнителя при непосредственном осуществлении демонтажа $j$-го излучающего элемента $\left(c_{j}^{(2)}\right.$ определяет статическую компоненту критерия). Упомянутые новые функции выражаются в терминах (3.4). В свою очередь, в рассматриваемом далее алгоритме определение значений функций (3.4) имеет следующий, весьма специальный, характер.

Мы исходим из того, что с каждым номером $j \in \overline{1, N}$ связывается помимо мегаполиса $M_{j}$ точка $\mu_{j} \in X$, характеризующая положение источника (в содержательной плоской задаче о демонтаже можно полагать, что $X=\mathbb{R} \times \mathbb{R}$ (плоскость), а $\mu_{j}$ - элемент выпуклой оболочки $M_{j}$; это отвечает идее представления мегаполиса как дискретизированной “границы” ближней зоны 
источника). Тогда при $x \in \mathfrak{M}_{j}$ число $c_{j}^{(1)}(x, K)$ определяется в реальном случае дозой радиации, полученной исполнителем при перемещении из $x$ к $\mu_{j}$ по прямой на расстояние несколько меньшее, чем (евклидово) расстояние между $x$ и $\mu_{j}$. Эта доза вычисляется по формулам, приведенным в [32]; она и рассматривается в качестве $c_{j}^{(1)}(x, k)$. При этом $c_{j}^{(1)}(x, k)$, где $k \in \overline{1, N}$, отражает (см. [32]) дозу, отвечающую перемещению из $x$ к $\mu_{j}$, при воздействии только одного источника с номером $k$. Тогда полагаем, что

$$
c_{j}^{(1)}(x, K) \triangleq \sum_{k \in K} \bar{c}_{j}^{(1)}(x, k) \quad \forall j \in \overline{1, N} \quad \forall x \in \mathfrak{M}_{j} \quad \forall K \in \mathfrak{N} .
$$

Относительно функций $c_{j}^{(2)}, j \in \overline{1, N}$, в $(3.4),(3.5)$ будем исходить из следующих предположений. Именно при $j \in \overline{1, N}$ и $k \in \overline{1, N}$ полагаем, что

$$
\bar{c}_{j}^{(2)}(k) \triangleq \gamma_{j, k} \Delta t_{j}
$$

где $\Delta t_{j} \in \mathbb{R}_{+}, \Delta t_{j}>0$, есть время, необходимое для демонтажа источника с номером $j$, а $\gamma_{j, k} \in \mathbb{R}_{+}, \gamma_{j, k}>0$, есть коэффициент, зависящий от интенсивности источника с номером $k$ и расстояния между источниками, имеющими номера $j$ и $k$. Мы полагаем при $j \in \overline{1, N}$ и $K \in \mathfrak{N}$, что $c_{j}^{(2)} \in \mathcal{R}_{+}[K]$ определяется условиями

$$
c_{j}^{(2)}(K) \triangleq \sum_{k \in K} \bar{c}_{j}^{(2)}(k) \quad \forall K \in \mathfrak{N} .
$$

Функция $c_{j}^{(2)}$ позволяет оценивать "множественную” дозу, получаемую исполнителем при демонтаже источника с номером $j$.

Наконец, введем в рассмотрение функцию $c_{j}^{(3)} \in \mathcal{R}_{+}\left[\mathbf{M}_{j} \times \mathfrak{N}\right]$, оценивающую дозу, получаемую исполнителем при его перемещении от источника $\mu_{j}$ (точнее, из некоторой его окрестности) к пункту отправления из мегаполиса $M_{j}$. Для этой цели используем функции из (3.4), (3.5). Относительно данных функций исходим из следующих предположений. Если $j \in \overline{1, N}, y \in \mathbf{M}_{j}$ и $k \in \overline{1, N} \backslash\{j\}$, то функция $\bar{c}_{j}^{(3)}(y, k)$ оценивает дозу, получаемую исполнителем от источника с номером $k$ при перемещении из окрестности этого источника к точке $y$ (конкретные значения указанной функции определяются формулами, приведенными в [32]). Кроме того, мы полагаем при $j \in \overline{1, N}$ и $y \in \mathbf{M}_{j}$, что $\bar{c}_{j}^{(3)}(y, j)=0$; имеется в виду, что на этом этапе перемещений исполнителя источник с номером $j$ уже демонтирован. Тогда при $j \in \overline{1, N}, y \in \mathbf{M}_{j}$ и $K \in \mathfrak{N}$

$$
c_{j}^{(3)}(y, K) \triangleq \sum_{k \in K} \bar{c}_{j}^{(3)}(y, k) .
$$

С учетом $(3.2),(3.4),(3.5),(3.8)$ и (3.9) получаем, что при $j \in \overline{1, N}, z \in \mathbb{M}_{j}$ и $K \in \mathfrak{N}$

$$
\begin{gathered}
c_{j}(z, K)=\sum_{k \in K}\left[\bar{c}_{j}^{(1)}\left(p r_{1}(z), k\right)+\bar{c}_{j}^{(2)}(k)+\bar{c}_{j}^{(3)}\left(p r_{2}(z), k\right)\right] \\
=\sum_{k \in K} \bar{c}_{j}^{(1)}\left(p r_{1}(z), k\right)+\sum_{k \in K} \bar{c}_{j}^{(2)}(k)+\sum_{k \in K} \bar{c}_{j}^{(3)}\left(p r_{2}(z), k\right) \\
=c_{j}^{(1)}\left(\operatorname{pr}_{1}(z), K\right)+c_{j}^{(2)}(K)+c_{j}^{(3)}\left(p r_{2}(z), K\right) .
\end{gathered}
$$

Заметим,что данная детализация (3.10) функций $c_{1}, \ldots, c_{n}$ относится к нашей конкретной задаче, ориентированной на проблему демонтажа излучающих элементов. Общая конструкция решения на основе ДП при любых функциях (2.9) содержится в [16] и в целом ряде других работ.

Полагаем в рассматриваемой конкретной задаче, что функция $f$ тождественно равна нулю; это отвечает ситуации, когда все радиационно опасные элементы демонтированы. 
3 а м е ч а н и е 1. Отметим одну альтернативную возможность, естественную для приложений. Речь идет о ситуации, когда количество "активных" источников настолько велико, что исполнитель (или исполнители) не в состоянии выполнить все работы по демонтажу; точнее, полученная (при исполнении намеченной части заданий) доза радиации такова, что работа по демонтажу должна быть прервана. В этом случае функция $f$ является ненулевой и определяет всякий раз дозу радиации, получаемую исполнителем при перемещении из последней "на маршруте" точки (см. $p r_{2}\left(z_{N}\right)$ в $\left.(2.10)\right)$ в упомянутую финишную точку. Предполагая такую возможность, мы будем рассматривать случай (2.10) с произвольной неотрицательной функцией $f$ (см. (2.9)). Излагаемая ниже конструкция является на самом деле алгоритмом на функциональном уровне.

\section{4. Динамическое программирование}

Мы кратко напомним общую схему (см. [14]) построения "алгоритмической версии" процедуры на основе ДП. Используем ниже оператор $\mathbf{I}$ (см. [15, часть 2]): $\mathbf{I} \in \mathfrak{N}^{\mathfrak{N}}$, и при $K \in \mathfrak{N}$

$$
\mathbf{I}(K) \triangleq K \backslash\left\{p r_{2}(z): z \in \Xi[K]\right\},
$$

где $\Xi[K] \triangleq\left\{z \in \mathbf{K} \mid\left(p r_{1}(z) \in K\right) \&\left(p r_{2}(z) \in K\right)\right\}$. Легко видеть, что $\mathbf{I}(\{t\})=\{t\} \forall t \in \overline{1, N}$. В самом деле, согласно $(2.7)(t, t) \notin \mathbf{K} \forall t \in \overline{1, N}$. Мы ограничимся поэтому построением слоев функции Беллмана. Для этого сначала введем, следуя $[14, \S 4.9]$, семейство всех существенных (в смысле реализации) ДП списков заданий:

$$
\mathfrak{G} \triangleq\left\{K \in \mathfrak{N} \mid \forall z \in \mathbf{K} \quad\left(p r_{1}(z) \in K\right) \Rightarrow\left(p r_{2}(z) \in K\right)\right\}
$$

(списки заданий суть непустые п/м $\overline{1, N}$ и только они). Данное семейство (4.1) допускает естественное разбиение в сумму $N$ подсемейств с ранжированием по мощности: $\mathfrak{G}_{s} \triangleq\{K \in$ $\mathfrak{G}|s=| K \mid\} \forall s \in \overline{1, N}$. Ясно, что $\mathfrak{G}_{N}=\{\overline{1, N}\}$ (синглетон, содержащий $\overline{1, N}$ ), и, кроме того,

$$
\mathfrak{G}_{1}=\left\{\{t\}: t \in \overline{1, N} \backslash \mathbf{K}_{1}\right\},
$$

где (здесь и ниже) $\mathbf{K}_{1} \triangleq\left\{p r_{1}(z): z \in \mathbf{K}\right\}$. Наконец (см. [15, (4.3)]),

$$
\mathfrak{G}_{s-1}=\left\{K \backslash\{t\}: K \in \mathfrak{G}_{s}, t \in \mathbf{I}(K)\right\} \quad \forall s \in \overline{2, N} .
$$

Таким образом, получаем реализуемую посредством (4.3) рекуррентную процедуру (см. [14, (4.4)]): начиная с $\{\overline{1, N}\}$ все семейства $\mathfrak{G}_{s}, s \in \overline{1, N}$, могут быть последовательно построены. Теперь строим слои пространства позиций: $D_{0}, D_{1}, \ldots, D_{N}$. Каждый слой является непустым п/м $\mathbf{X} \times \mathcal{P}(\overline{1, N})$.

Пусть $\widetilde{\mathcal{M}}$ - объединение всех множеств $\mathbf{M}_{j}, j \in \overline{1, N} \backslash \mathbf{K}_{1}$. Тогда полагаем

$$
D_{0} \triangleq\{(x, \varnothing): x \in \widetilde{\mathcal{M}}\}, \quad D_{N} \triangleq\left\{(x, \overline{1, N}): x \in X^{0}\right\} .
$$

Если $s \in \overline{1, N-1}$ и $K \in \mathfrak{G}_{s}$, то (см. [14, (4.5)]) вводим последовательно множества

$$
\begin{gathered}
J_{s}(K) \triangleq\left\{j \in \overline{1, N} \backslash K \mid\{j\} \cup K \in \mathfrak{G}_{s+1}\right\}, \\
\mathcal{M}_{s}[K] \triangleq \bigcup_{j \in J_{s}(K)} \mathbf{M}_{j}, \quad \mathbb{D}_{s}[K] \triangleq\left\{(x, K): x \in \mathcal{M}_{s}[K]\right\} .
\end{gathered}
$$

В этих терминах определяется при $s \in \overline{N-1}$ слой $D_{s}$ :

$$
D_{s} \triangleq \bigcup_{K \in \mathfrak{G}_{s}} \mathbb{D}_{s}[K]
$$


Множества-слои связаны следующим соотношением (см. [14, (4.11)]):

$$
\left(\operatorname{pr}_{2}(z), K \backslash\{j\}\right) \in D_{s-1} \quad \forall s \in \overline{1, N} \quad \forall(x, K) \in D_{s} \quad \forall j \in \mathbf{I}(K) \quad \forall z \in \mathbb{M}_{j}
$$

Следующий этап - построение слоев функции Беллмана: речь идет о последовательном построении функций

$$
v_{0} \in \mathcal{R}_{+}\left[D_{0}\right], v_{1} \in \mathcal{R}_{+}\left[D_{1}\right], \ldots, v_{N} \in \mathcal{R}_{+}\left[D_{N}\right] .
$$

Мы используем здесь [14, (3.10), замечание 3.1]. Итак, построение функций (4.6) осуществляется по схеме $[14,(4.13)]$. Прежде всего мы полагаем, что $v_{0}$ в (4.6) находится по правилу, учитывающему первое положение в (4.4):

$$
v_{0}(x, \varnothing) \triangleq f(x) \quad \forall x \in \widetilde{\mathcal{M}} .
$$

Если же $s \in \overline{1, N}$ и функция $v_{s-1} \in \mathcal{R}_{+}\left[D_{s-1}\right]$ уже построена, то определяем $v_{s} \in \mathcal{R}_{+}\left[D_{s}\right]$ по следующему правилу (учитывающему (4.5)): при $(x, K) \in D_{s}$

$$
v_{s}(x, K) \triangleq \min _{j \in \mathbf{I}(K)} \min _{z \in \mathbb{M}_{j}}\left[\mathbf{c}\left(x, p r_{1}(z), K\right)+c_{j}(z, K)+v_{s-1}\left(p r_{2}(z), K \backslash\{j\}\right)\right]
$$

используем в (4.8) тот факт, что согласно (4.5) известны значения

$$
v_{s-1}\left(p_{2}(z), K \backslash\{j\}\right) \in \mathbb{R}_{+} \quad \forall j \in \mathbf{I}(K) \quad \forall z \in \mathbb{M}_{j} .
$$

Тем самым определено правило преобразования $v_{s-1}$ в $v_{s}$ при $s \in \overline{1, N}$. В частности, $v_{N-1}$ преобразуется в $v_{N}$ по правилу (см. (4.4), (4.8)): при $x \in X^{0}$

$$
v_{N}(x, \overline{1, N})=\min _{j \in \mathbf{I}(\overline{1, N})} \min _{z \in \mathbb{M}_{j}}\left[\mathbf{c}\left(x, p r_{1}(z), \overline{1, N}\right)+c_{j}(z, \overline{1, N})+v_{N-1}\left(p r_{2}(z), \overline{1, N} \backslash\{j\}\right)\right] .
$$

Предложение 1. Если $x \in X^{0}, m o \widetilde{V}[x]=v_{N}(x, \overline{1, N})$.

Д о к а з а т е л ь с т в о следует из того факта, что каждая функция (4.6) является сужением "единой" (для всех $x \in X^{0}$ ) функции Беллмана, а тогда мы используем представление $[14,(3.10)]$ (см. также [33, (4.5)]). В этой связи заметим, что каждое преобразование (4.8) определяется на самом деле уравнением Беллмана.

Итак, цепочка $v_{0} \longrightarrow v_{1} \longrightarrow \ldots \longrightarrow v_{N}$ позволяет построить функцию

$$
\widetilde{V}[\cdot] \triangleq(\widetilde{V}[x])_{x \in X^{0}}
$$

и, стало быть, найти $\mathbb{V}(2.13)$, а также множество

$$
X_{\text {opt }}^{0} \triangleq\left\{x^{0} \in X^{0} \mid \widetilde{V}\left[x^{0}\right]=\mathbb{V}\right\} \neq \varnothing .
$$

Для наших целей, однако, достаточно определить какую-либо одну точку $x_{0} \in X_{o p t}^{0}$. Будем считать это - выполненным и данную точку $x_{0}$ фиксируем до конца раздела.

Рассмотрим теперь процедуру построения (оптимального) решения из множества (2.14). На этом этапе сначала выбираем решение из множества $(\mathrm{sol})\left[x_{0}\right]$, что соответствует $[14, \S 6]$, а именно мы используем процедуру ДП (см. [14, (6.1)-(6.5)]). В итоге получаем (оптимальное) ДР

$$
\left(\eta,\left(\mathbf{z}^{(j)}\right)_{j \in \overline{0, N}}\right) \in(\mathrm{sol})\left[x_{0}\right]
$$

задачи (2.11) при $x=x_{0}$. Теперь уже вполне очевидно следующее предложение.

Предложение 2. Триплет $\left(\eta,\left(\mathbf{z}^{(j)}\right)_{j \in \overline{0, N}}, x_{0}\right)$ является оптимальным решением задачи (2.12) :

$$
\left(\eta,\left(\mathbf{z}^{(j)}\right)_{j \in \overline{0, N}}, x_{0}\right) \in \mathbf{S O L} .
$$


Д о к а з а т е л ь с т в о выстраиваем как непосредственную комбинацию (4.11) и (4.12): по выбору $x_{0}$ имеем

$$
\mathfrak{G}_{\eta}\left[\left(\mathbf{z}^{(j)}\right)_{j \in \overline{0, N}}\right]=\widetilde{V}\left[x_{0}\right]=\mathbb{V},
$$

а тогда (4.13) вытекает из (2.14), поскольку $\left(\eta,\left(\mathbf{z}^{(j)}\right)_{j \in \overline{0, N}}, x_{0}\right) \in \mathbf{D}$ согласно (2.8) (действительHо, $\left(\eta,\left(\mathbf{z}^{(j)}\right)_{j \in \overline{0, N}}\right) \in \widetilde{\mathbf{D}}\left[x_{0}\right]$, a $\left.x_{0} \in X^{0}\right)$.

3 а м е ч а н и е 2. Отметим одну модификацию общей процедуры на основе ДП, имея в виду возможность, отмеченную (для другой задачи) в [34]. Речь идет о ситуации, когда нам требуется найти только экстремум $\mathbb{V}$ и множество (4.11). В этом случае построение функций (4.6) может осуществляться с некоторой экономией памяти вычислителя. Совсем кратко напомним данную модификацию.

Итак, вернемся к этапу, когда построены все слои $D_{0}, D_{1}, \ldots, D_{N}$ пространства позиций. Функция $v_{0}$ определяется по-прежнему (см. (4.7)). Пусть вообще $s \in \overline{1, N}$ и функция $v_{s-1}$ уже построена. Тогда, располагая только функцией $v_{s-1}$, мы по правилу (4.8) конструируем $v_{s} \in \mathcal{R}_{+}\left[D_{s}\right]$. Если $s=N$, то построение завершено. Если же $s<N$, то массив значений $v_{s-1}$ уничтожается и заменяется массивом значений $v_{s}$ в интересах построения $v_{s+1}$, где $s+1 \in \overline{1, N}$. При этом построении используется аналог (4.8) при замене (в (4.8)) $s-1$ на $s$ и $s$ на $s+1$. Данная процедура после $N$ этапов указанного типа реализует $v_{N}$, и мы снова можем использовать предложение 1 для нахождения $\widetilde{V}[\cdot](4.10)$ и, как следствие, для определения $\mathbb{V}$ и $X_{o p t}^{0}(4.11)$. В свою очередь $\mathbb{V}$ и $X_{\text {opt }}^{0}$ могут использоваться (в задачах большой размерности) для тестирования эвристик.

\section{5. Алгоритм на функциональном уровне}

При исследовании задачи о демонтаже системы радиоционно опасных элементов, и в частности в задаче, моделирующей процесс демонтажа энергоблока АЭС, выведенного из эксплуатации, серьезную проблему составляет построение функций (2.9), обсуждавшееся на идейном уровне в разд. 3 (см. в частности, (3.1), (3.2), (3.6)-(3.10)). Данное построение подробно исследовалось также в [32], и мы сейчас не будем его рассматривать, отметив, однако, некоторые возможности, связанные с применением ДП и проявляющиеся в (4.7)-(4.9) (имеется в виду возможность насчитывания функций (2.9) "частями" по ходу построения слоев функций Беллмана). Сейчас будем полагать кортеж (2.9) построенным.

Этап построения существенных списков заданий. Располагая $\mathfrak{G}_{N}=\{\overline{1, N}\}$, реализуем процедуру

$$
\mathfrak{G}_{N} \longrightarrow \mathfrak{G}_{N-1} \longrightarrow \ldots \longrightarrow \mathfrak{G}_{1}
$$

оперируя построениями (4.2), (4.3).Итогом является построение разбиения семейства (4.1).

Этап построения слоев пространства позиций. Располагая семействами $\mathfrak{G}_{1}, \ldots, \mathfrak{G}_{N}$, конструируем непустые множества $D_{0}, D_{1}, \ldots, D_{N}$. Слой $D_{0}$ определяется явно (см. (4.4)). Для построения $D_{s}$ при $s \in \overline{1, N-1}$ последовательно конструируются множества $J_{s}(K), \mathcal{M}_{s}[K]$ и $\mathbb{D}_{s}[K]$, где $K \in \mathfrak{G}_{s}$; слой $D_{s}$ получаем объединением всех $\mathbb{D}_{s}[K], k \in \mathfrak{G}_{s}$. Слой $D_{N}$ определяется явно (см. (4.4)). Итогом этапа является кортеж $\left(D_{s}\right)_{s \in \overline{0, N}}$ со свойством (4.5).

Этап построения слоев функиии Беллмана. Имеются два варианта:

1) Построение глобального экстремума и множества (4.11) всех оптимальных точек старта (возможна модификация, когда требуется найти какую-либо точку из множества (4.11)). Логика процедуры соответствует приведенному выше замечанию.

2 ) Полное решение задачи (2.12), когда наряду с $\mathbb{V}(2.13)$ определяется какое-либо решение из множества (2.14). В этом случае итогом данного этапа является кортеж $\left(v_{0}, v_{1}, \ldots, v_{n}\right)$, 
элементами которого выступают функции (4.6). При этом функция $v_{0}$ задается явно (см. (4.7)). Далее реализуется рекуррентная процедура

$$
v_{0} \longrightarrow v_{1} \longrightarrow \ldots \longrightarrow v_{N}
$$

для нее преобразование $v_{s-1}$ в $v_{s}$ при $s \in \overline{1, N}$ задается правилом (4.8), которое использует (4.5). При этом функции, получающиеся при реализации (5.1), сохраняются в памяти вычислителя. В частности, мы определяем $v_{N}$ и, стало быть, находим $\widetilde{V}[\cdot]$ (см. предложение 1 ) как функцию на $X^{0}$. Кроме того, определяем простым перебором точку $x_{0} \in X_{o p t}^{0}$, которая выполняет роль точки старта.

Этап построения оптимального решения. Располагая кортежем $\left(v_{0}, v_{1}, \ldots, v_{N}\right)$, реализуем процедуру $[14,(6.1)-(6.5)]$ для построения ДР (4.12). На этом этапе существенно используются слои функции Беллмана (см. (4.6)). В варианте $[14,(6.1)-(6.5)]$ в качестве точки старта определяем $x_{0}$, найденное на предыдущем этапе. Согласно предложению 2 в виде (4.13) получаем искомое оптимальное решение задачи (2.12). Напомним, что процедура $[14,(6.1)-(6.5)]$ (см. также $[16 ; 32 ; 33])$ предусматривает "одновременное" построение маршрута и траектории, что очевидно уже из (4.8) (соотношения (4.8) являются базовыми для вышеупомянутых конструкций $[14 ; 16 ; 32 ; 33]$ на основе ДП).

\section{6. Вычислительный эксперимент}

Рассматриваемые в настоящей работе алгоритмические конструкции были реализованы в виде программы для ПЭВМ, написанной на языке программирования $\mathrm{C}++$ и работающей под управлением 64-разрядной операционной системы семейства Windows, начиная c Windows 7. Методология подсчета получаемой исполнителем дозы подробно рассмотрена в разд. 3 (см. выше), при этом используются формулы, выведенные в работе [32] для вычисления интегралов в выражениях функций стоимости (3.4)-(3.10). Вычислительная часть программы реализована в отдельном от интерфейса пользователя потоке. Для случая решения задачи на плоскости имеется возможность графического представления траектории посещения мегаполисов, а также увеличения отдельных участков графика и сохранения изображения в файл графического формата bmp. Исходные данные, а также результаты счета программы хранятся в текстовом файле специальной структуры.

Вычислительный эксперимент проводился на ПЭВМ с центральным процессором Intel Core i7 объемом оперативной памяти 64 гБ, с установленной операционной системой Windows 7 Максимальная SP1. Рассмотрим модельный пример, в котором на плоскости задан 31 мегаполис. Каждый мегаполис $M_{i}, i \in \overline{1,31}$, представляет собой равномерную "сетку" на окружности или прямоугольнике, а точка $\mu_{i}$, символизирующая источник радиации, расположена в выпуклой оболочке мегаполиса; элементы множества $X^{0}$ находятся за пределами соответствующей фигуры (прямоугольника или окружности). При вычислении значений функций стоимости (3.4)-(3.10) по формулам, приведенным в [32], используется евклидово расстояние.

Пусть множество начальных состояний имеет вид

$$
\left.X^{0}=\{(-15,-120) ;(0,0) ;(90,55) ;(-90,-80) ;(-90,90) ;(30,-30))\right\} .
$$

Количество адресных пар, определяющих ограничения на порядок посещения мегаполисов, равно $34(|\mathbf{K}|=34)$. Интенсивности излучения источников $\gamma_{i, k}, i \in \overline{1,31}, k \in \overline{1,31}$ находятся в пределах от 1.3 до 5.5. Длительности работ по демонтажу источников $\Delta t_{j}, j \in \overline{1,31}$, составляют от 1.1 до 1.8 (в зависимости от источника). Радиусы ближних зон источников излучения (ограничения на приближение к источнику при демонтаже) также различны и находятся в пределах от 1.0 до 1.6. 


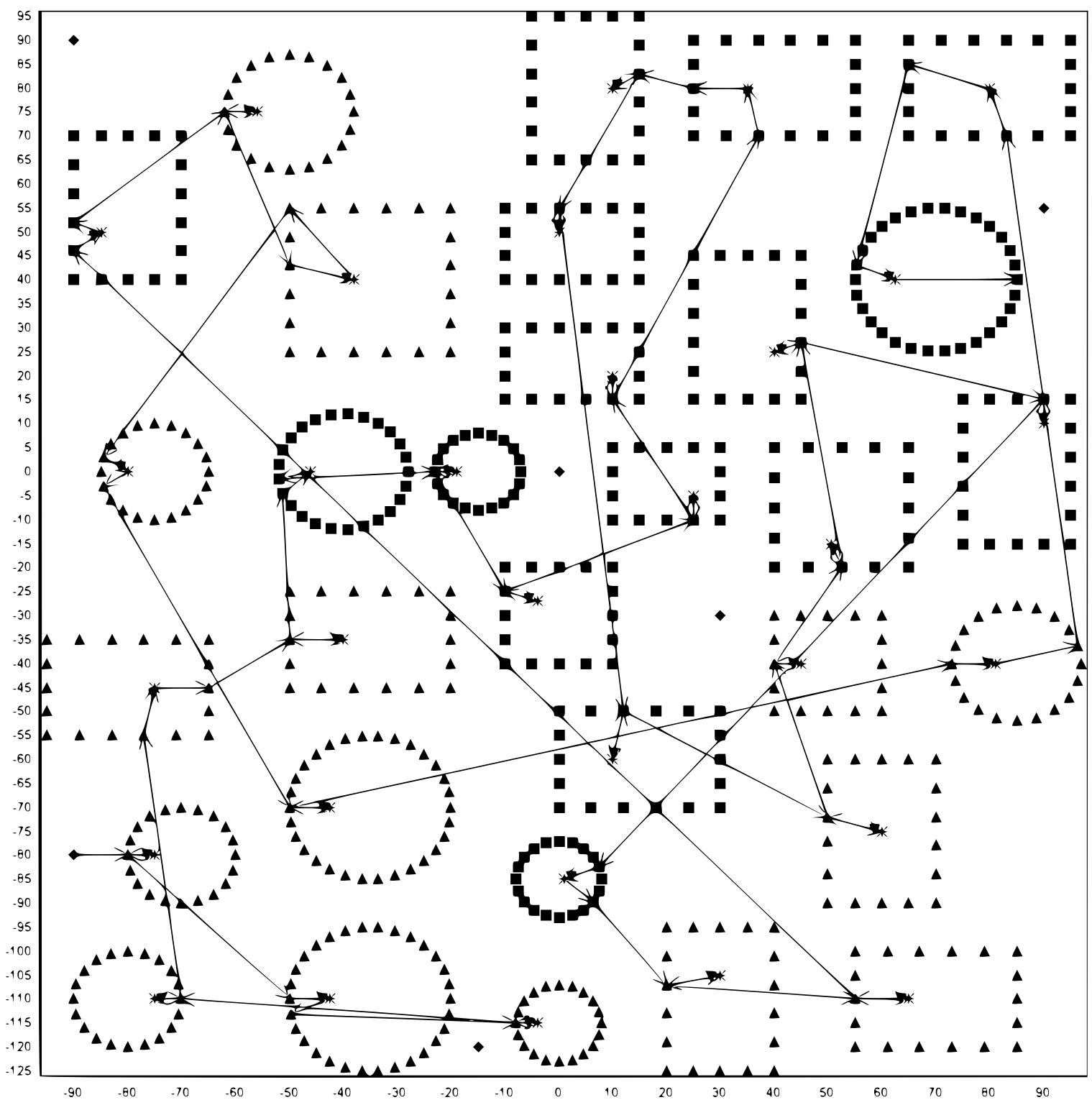

Траектория движения по мегаполисам

Получены следующие результаты:

величина совокупных затрат $\mathbb{V}=183.78$;

начальный пункт обхода мегаполисов $(-90,-80)$;

финальный пункт посещения мегаполисов - УП $(55.33,43.12 ; 85,40) \in M_{16} \times M_{16}$.

Графики маршрута и трассы приведены на рисунке (с помощью значков $*$ отображаются источники излучения, $\diamond-$ элементы множества $X^{0}$, а $\nabla-$ позиции выполнения работ по демонтажу в окрестности источников излучения).

Время счета составило 11 час. 42 мин. 37 сек.

\section{СПИСОК ЛИТЕРАТУРЫ}

1. Gutin G., Punnen A.P. The traveling salesman problem and its variations. Berlin: Springer, 2002. $850 \mathrm{p}$.

2. Cook W. J. In pursuit of the traveling salesman: Mathematics at the limits of computation. Princeton: Princeton University Press, 2012. 228 p.

3. Гимади Э. Х., Хачай М. Ю. Экстремальные задачи на множествах перестановок. Екатеринбург: Изд-во УМЦ УПИ, 2016. 220 с. 
4. Меламед И. И., Сергеев С. И., Сигал И. Х. Задача коммивояжера. Вопросы теории // Автоматика и телемеханика. 1989. №9. С. 3-34.

5. Меламед И.И., Сергеев С.И., Сигал И.Х. Задача коммивояжера. Точные алгоритмы // Автоматика и телемеханика. 1989. №10. С. 3-29.

6. Меламед И. И., Сергеев С. И., Сигал И. Х. Задача коммивояжера. Приближенные алгоритмы // Автоматика и телемеханика. 1989. №11. С. 3-26.

7. Беллман Р. Применение динамического программирования к задаче о коммивояжере // Кибернетический сб. М.: Мир. 1964. Т. 9. С. 219-228.

8. Хелд М., Карп Р. М. Применение динамического программирования к задачам упорядочения // Кибернетический сб. М.: Мир. 1964. Т. 9. С. 202-218.

9. Литл Дж., Мурти К., Суини Д., Кэрел К. Алгоритм для решения задачи о коммивояжере // Экономика и мат. методы. 1965. Т. 1 (Вып. 1). С. 94-107.

10. Коробкин В.В., Сесекин А.Н., Ташлыков О.Л., Ченцов А.Г. Методы маршрутизации и их приложения в задачах повышения безопасности и эффективности эксплуатации атомных станций / под общ. ред. чл.-кор. РАН И. А. Каляева. Москва: Новые технологии, 2012. 234 с.

11. Bianco L., Mingozzi A., Ricciardelli S. et.al. The Traveling salesman problem with precedence constraints // Papers of the 19th Annual Meeting / eds. W. Bühler, G. Feichtinger, R.F. Hartl, F.J. Radermacher, P. Stähly, Vorträge der 19. Jahrestagung. Berlin: Springer, 1992. P. 299-306. (Operations Research Proceedings; vol. 1990.) doi: 10.1007/978-3-642-77254-2_33.

12. Castclino K., D'Souza R., Wright P.K. Toolpath optimization for minimizing airtime during machining // J. Manufacturing Systems. 2003. Vol. 22, №3. P. 173-189. doi: 10.1016/S0278-6125(03)90018-5 .

13. Salii Ya. Order-theoretic characteristics and dynamic programming for precedence constrained traveling salesman problem // Turku Center for Computer Science. 2017. Vol. 26. (Proc. of the 4th Russian Finnish Symposium on Discrete Mathematics, Turku, May 16-19 2017). P. 152-164.

14. Chentsov A.G., Chentsov P. A. The routing problems with optimization of the starting point: dynamic programming // Изв. Ин-та математики и информатики Удмурт. гос. ун-та. 2019. Т. 54. C. $102-121$.

15. Ченцов А. Г. Экстремальные задачи маршрутизации и распределения заданий: вопросы теории. Москва; Ижевск: НИЦ "Регулярная и хаотическая динамика", 2008. 238 с.

16. Ченцов А.Г., Ченцов А. А. Задача маршрутизации, осложненная зависимостью функций стоимости и "текущих" ограничений от списка заданий // Моделирование и анализ информационных систем. 2016. Т. 23, №2. С. 211-227.

17. Grigoryev A. M., Tashlykov O.L. Solving a routing optimization of works in radiation fields with using a supercomputer // AIP Conf. Proc. Vol. 2015. Art-no. 020028. 2018. doi: 10.1063/1.5055101.

18. Grigoryev A.M., Tashlykov O.L. Route optimization during works in non-stationary radiation fields with obstacles // AIP Conf. Proc. Vol. 2174 (1). Art-no. 020216. 2019. doi: 10.1063/1.5134367.

19. Кропачев Ю.А., Ташлыков О.Л., Щеклеин С. Е. Оптимизация радиационной защиты на этапе вывода энергоблоков АЭС из эксплуатации // Изв. вузов. Ядерная энергетика. 2019. №1. C. $119-130$.

20. Ташлыков О. Л., Щеклеин С. Е., Лукьяненко В. Ю., Михайлова А.Ф., Русских И. М., Селезнев Е. Н., Козлов А. В. Оптимизация состава радиационной защиты // Изв. вузов. Ядерная энергетика. 2015. №4. С. 36-42.

21. Коробкин В. В., Ченцов П. А. Оптимизация перемещения и замены тепловыделяющих сборок в активной зоне реактора типа ВВЭР / Мехатроника, автоматизация, управление МАУ-2009 // Материалы Междунар. науч.-техн. конф. Таганрог: ТТИ ЮФУ. 2009. С. 347-349.

22. Bohez E., Makhanov S. S., Sonthipermpoon K. Adaptive nonlinear tool path optimization for five-axis machining // Internat. J. Product. Research. 2000. Vol. 38, no. 17. P. 4329-4343.

23. Dewil R., Vansteenwegen P., Cattrysse D. Construction heuristics for generating tool paths for laser cutters // Internat. J. Product. Research. 2014. Vol. 52, no. 20. P. 5965-5984.

24. Dewil R., Vansteenwegen P., Cattrysse D. An improvement heuristic framework for the laser cutting tool path problem // Internat. J. Product. Research. 2015. Vol. 53, no. 6. P. 1761-1776.

25. Dewil R., Vansteenwegen P., Cattrysse D. A review of cutting path algorithms for cutters // Internat. J. Advanced Manufactoring Technology. 2016. Vol. 87. P. 1865-1884.

26. Petunin A. A. Modelling of tool path for the CNC Sheet Cutting Machines // AIP Conf. Proceedings. 2015. Vol. 1690. P. 060002(1)-060002(7). 
27. Petunin A. A., Stylios S. Optimization models of tool path problem for CNC Sheet Metal Cutting Machines // IFAC - PaperOnLine. 2016. Vol. 49, no. 12. P. 23-28.

28. Petunin A. General model of tool path problem for the CNC Sheet Cutting Machines // IFAC PaperOnLine. 2019. Vol. 52, no. 13. P. 2662-2667.

29. Lee M.-K., Kwon K.-B. Cutting path optimization in CNC cutting processes using a two-step genetic algorithm // Internat. J. Product. Research. 2006. Vol. 44, no. 24. P. 5307-5326.

30. Куратовский К., Мостовский А. Теория множеств. М.: Мир, 1970. 416 с.

31. Дьедонне Ж. Основы современного анализа. М.: Мир, 1964. 430 с.

32. Ченцов А.А., Ченцов А.Г. Модельный вариант задачи о последовательной утилизации источников излучения (итерации на основе оптимизирующих вставок) // Изв. Ин-та математики и информатики Удмурт. гос. ун-та. 2017. Т. 50. С. 83-109. doi: 10.20537/2226-3594-2017-50-08 .

33. Ченцов А.Г., Ченцов А.А. Задача маршрутизации с ограничениями, зависящими от списка заданий // Докл. РАН. 2015. Т. 465, № 2. С. 154-158.

34. Lawler E. L. Efficient implementation of dynamic programming algorithms for sequencing problems: Tech. Rep.: BW 106/79: Stichting Mathematisch Centrum. 1979. Vol. 106, no. 79. P. 1-16.

Поступила 21.06.2020

После доработки 21.07.2020

Принята к публикации 10.08.2020

Ченцов Александр Георгиевич

д-р физ.-мат. наук, профессор, чл.-кор. РАН

главный науч. сотрудник

Институт математики и механики им. Н. Н. Красовского УрО РАН;

профессор

Уральский федеральный университет им. Б. Н. Ельцина,

г. Екатеринбург

e-mail: chentsov@imm.uran.ru

Ченцов Алексей Александрович

канд. физ.-мат. наук

научн. сотрудник

Институт математики и механики им. Н.Н. Красовского УрО РАН

г. Екатеринбург

e-mail: chentsov.a@binsys.ru

Сесекин Александр Николаенвич

д-р физ.-мат. наук, профессор

зав. кафедрой прикладной математики и механики

Уральский федеральный университет;

ведущий научн. сотрудник

Институт математики и механики им. Н.Н. Красовского УрО РАН

г. Екатеринбург

e-mail: a.n.sesekin@urfu.ru

\section{REFERENCES}

1. Gutin G., Punnen A.P. The traveling salesman problem and its variations. Berlin: Springer, 2002, 850 p. ISBN: 0-306-48213-4.

2. Cook W.J. In pursuit of the traveling salesman: mathematics at the limits of computation. Princeton University Press, 2012, 228 p. ISBN: 0691152705.

3. Gimadi E.Kh., Khachai M.Yu. Ekstremal'nye zadachi na mnozhestvakh perestanovok [Extremal problems on sets of permutations] Yekaterinburg: UMC UPI Publ., 2016, 220 p. ISBN 978-5-8295-0497-7.

4. Melamed I.I., Sergeev S.I., Sigal I.Kh. The traveling salesman problem. Issues in theory. Autom. Remote Control, 1989, vol. 50, no. 9, pp. 1147-1173. 
5. Melamed I.I., Sergeev S.I., Sigal I.Kh. The traveling salesman problem. Exact methods. Autom. Remote Control, 1989, vol. 50, no. 10, pp. 1303-1324.

6. Melamed I.I., Sergeev S.I., Sigal I.Kh. The traveling salesman problem. Approximate algorithms. Autom. Remote Control, 1989, vol. 50, no. 11, pp. 1459-1479.

7. Bellman R. Dynamic programming treatment of the travelling salesman problem. J. ACM, 1962, vol. 9, no. 1, pp. 61-63. doi: 10.1145/321105.321111.

8. Held M., Karp R.M. A dynamic programming approach to sequencing problems. Journal of the Society for Industrial and Applied Mathematics, 1962, vol. 10, no. 1, pp. 196-210. doi: 10.1137/0110015.

9. Little L.D.C., Murty K.G., Sweeney D.W., Karel C. An algorithm for the traveling salesman problem. Operations Research, 1963, vol. 11, no. 6, pp. 972-989. doi: 10.1287/opre.11.6.972 .

10. Korobkin V.V., Sesekin A.N., Tashlykov O.L., Chentsov A.G. Metody marshrutizatsii i ikh prilozheniya v zadachakh povysheniya bezopasnosti $i$ effektivnosti ekspluatatsii atomnykh stantsii [Routing methods and their applications in problems of the enhancement of safety and efficiency of nuclear plant operation]. Moscow: Novye Tekhnologii Publ., 2012, 234 p. ISBN: 97978-5-94694-027-6.

11. Bianco L., Mingozzi A., Ricciardelli S. et al. The Traveling salesman problem with precedence constraints. In: Bühler W., Feichtinger G., Hartl R.F., Radermacher F.J., Stähly P. (eds), Papers of the 19th Annual Meeting / Vorträge der 19. Jahrestagung, Operations Research Proceedings, vol. 1990, Berlin: Springer, 1992, pp. 299-306. doi: 10.1007/978-3-642-77254-2_33.

12. Castclino K., D'Souza R., Wright P.K. Toolpath optimization for minimizing airtime during machining. J. Manufacturing Systems, 2003, vol. 22, no. 3, pp. 173-180. doi: 10.1016/S0278-6125(03)90018-5.

13. Salii Ya. Order-theoretic characteristics and dynamic programming for precedence constrained traveling salesman problem. In: Proc. of the 4th Russian Finnish Symposium on Discrete Mathematics (Turku, May 16-19 2017), TUCS Lecture Notes, no. 26, pp. 152-164.

14. Chentsov A.G., Chentsov P.A. The routing problems with optimization of the starting point: dynamic programming. Izv. IMI UdGU, 2019, vol. 54, pp. 102-121. doi: 10.20537/2226-3594-2019-54-08 .

15. Chentsov A.G. Ekstremal'nye zadachi marshrutizatsii i raspredeleniya zadanii: voprosy teorii [Extremal problems of routing and distribution of tasks: questions of theory]. Moscow; Izhevsk: RHD Pibl., 2008, 238 p. ISBN: 978-5-93972-654-2 .

16. Chentsov A.G., Chentsov A.A. The routing problem complicated by the dependence of cost functions and "current" restrictions on the list of tasks. Modelirovanie i analiz informazionnikh sistem, 2016, vol. 23, no. 2, pp. 211-227 (in Russian). doi: 10.18255/1818-1015-2016-2-211-227.

17. Grigoryev A.M., Tashlykov O.L. Solving a routing optimization of works in radiation fields with using a supercomputer. AIP Conf. Proc., 2018, vol. 2015, art.-no. 020028. doi: 10.1063/1.5055101.

18. Grigoryev A.M., Tashlykov O.L. Route optimization during works in non-stationary radiation fields with obstacles. AIP Conf. Proc., 2019, vol. 2174 (1), art.-no. 020216. doi: 10.1063/1.5134367.

19. Kropachev, Y.A., Tashlykov, O.L., Shcheklein, S.E. Optimization of radiation protection at the NPP unit decommissioning stage. Izvestiya Wysshikh Uchebnykh Zawedeniy. Yadernaya Energetika, 2019, vol. 2019, pp. 119-130. doi: 10.26583/npe.2019.1.11.

20. Tashlykov, O.L., Shcheklein, S.E., Luk'yanenko, V.Y., Mikhailova A.F., Seleznyov, E.N., Kozlov, A.V. The optimization of radiation protection composition. Nuclear Energy and Technology, 2016, vol. 2, no. 1, pp. 42-44. doi: 10.1016/j.nucet.2016.03.008.

21. Korobkin V.V., Chentsov P.A. Optimization of the movement and replacement of fuel assemblies in the core of a WWER type reactor. In: Abstr. int. sci. tech. conf. "Mechatronics, automation, control" (MAC-2009). Taganrog: TTU SFU, 2009, pp. 347-349. ISBN: 978-5-8327-0339-8.

22. Bohez E., Makhanov S.S., Sonthipermpoon K. Adaptive nonlinear tool path optimization for five-axis machining. Internat. J. Product. Research, 2000, vol. 38, no. 17, pp. 4329-4343. doi: $10.1080 / 00207540050205127$.

23. Dewil R., Vansteenwegen P., Cattrysse D. Construction heuristics for generating tool paths for laser cutters. Internat. J. Produc. Research, 2014, vol. 52, no. 20, pp. 5965-5984. doi: 10.1080/00207543.2014.895064.

24. Dewil R., Vansteenwegen P., Cattrysse D. An improvement heuristic framework for the laser cutting tool path problem. Internat. J. Produc. Research, 2015, vol. 53, no. 6, pp. 1761-1776. doi: $10.1080 / 00207543.2014 .959268$.

25. Dewil R., Vansteenwegen P., Cattrysse D. A review of cutting path algorithms for laser cutters. Int. J. Adv. Manuf. Technol., 2016, vol. 87, no. 5, pp. 1865-1884. doi: 10.1007/s00170-016-8609-1. 
26. Petunin A.A. Modelling of tool path for the cnc sheet cutting machines. AIP Conf. Proc., 2015, vol. 1690, art.-no. 060002, 7 p. doi: 10.1063/1.4936740 .

27. Petunin A.A., Stylios S. Optimization models of tool path problem for cnc sheet metal cutting machines. IFAC-PapersOnLine, 2016, vol. 49, no. 12, pp. 23-28. doi: 10.1016/j.ifacol.2016.07.544 .

28. Petunin A. general model of tool path problem for the cnc sheet cutting machines. IFAC-PapersOnLine, 2019, vol. 52, no. 13, pp. 2662-2667. doi: 10.1016/j.ifacol.2019.11.609.

29. Lee M.-K., Kwon K.-B. Cutting path optimization in CNC cutting processes using a two-step genetic algorithm. Internat. J. Produc. Research, 2006, vol. 44, no. 24, pp. 5307-5326. doi: $10.1080 / 00207540600579615$.

30. Kuratowski K., Mostowski A. Set theory. Warszawa: PWN - Polish Scientific Publishers, 1968, 417 p. ISBN: 9780444534170 . Translated to Russian under the title Teoriya mnozhestv, Moscow: Mir Publ., 1970, $416 \mathrm{p}$.

31. Dieudonné J. Foundations of modern analysis. N Y: Acad. Press, 1960, 361 p. Translated to Russian under the title Osnovy sovremennogo analiza, Moscow: Mir Publ., 1964, 430 p.

32. Chentsov A.G., Chentsov A.A. A model variant of the problem about radiation sources utilization (iterations based on optimization insertions). Izv. IMI UdGU, 2017, vol. 50, pp. 83-109 (in Russian). doi: $10.20537 / 2226-3594-2017-50-08$.

33. Chentsov A.G., Chentsov A.A. Route problem with constraints depending on a list of tasks. Dokl. Math., 2015, vol. 92, no. 3, pp. 685-688. doi: 10.1134/S1064562415060083.

34. Lawler E.L. Efficient implementation of dynamic programming algorithms for sequencing problems. CWI Technical report. Stichting Mathematisch Centrum. Mathematische Besliskunde-BW, 1979, vol. 106, no. 79 , pp. $1-16$.

Received June 21, 2020

Revised July 21, 2020

Accepted August 10, 2020

Funding Agency: This work was supported by the Russian Foundation for Basic Research (project no. 19-01-00573) and is a part of the research carried out at the Ural Mathematical Center.

Alexander Georgievich Chentsov, Dr. Phys.-Math. Sci., Prof., Corresponding Member RAS. Krasovskii Institute of Mathematics and Mechanics of the Ural Branch of the Russian Academy of Sciences, Yekaterinburg, 620108 Russia; Ural Federal University Yekaterinburg, 620083 Russia, e-mail: chentsov@imm.uran.ru .

Aleksei Aleksandrovich Chentsov, Candidate of Phys.-Math. Sci. Krasovskii Institute of Mathematics and Mechanics of the Ural Branch of the Russian Academy of Sciences, Yekaterinburg, 620108 Russia, e-mail: chentsov.a@binsys.ru .

Alexander Nikolaevich Sesekin, Dr. Phys.-Math. Sci., Prof., Head of the Department of Applied Mathematics and Mechanics - Ural Federal University, Yekaterinburg, 620083 Russia; Krasovskii Institute of Mathematics and Mechanics of the Ural Branch of the Russian Academy of Sciences, Yekaterinburg, 620108 Russia, e-mail: a.n.sesekin@urfu.ru .

A. G. Chentsov, A. A. Chentsov, A.N. Sesekin. On the problem of sequential traversal of megalopolises with precedence conditions and cost functions depending on a list of tasks, Trudy Instituta Matematiki i Mekhaniki URO RAN, 2020, vol. 26, no. 3, pp. 219-234. 